\title{
Symplectic 4-manifolds, Stein domains, Seiberg-Witten theory and mapping class groups
}

\author{
ANDRÁS I STIPSICZ
}

In this survey we review some aspects of the connection between mapping class groups, symplectic 4-manifolds, Stein domains and Lefschetz fibrations on them.

57R17; 57R57

\section{Introduction}

Mapping class groups and their study play a central role in low-dimensional topology. Their connection to 3-manifold topology (through Heegaard decompositions, for example) is rather obvious; more recently (through open book decompositions), mapping class group questions arose in 3-dimensional contact topology.

It is less transparent how mapping class groups are related to 4-dimensional topology. By results of Donaldson and Gompf, closed symplectic manifolds (admitting Lefschetz fibration or Lefschetz pencil structures) give rise to various objects in mapping class groups, and therefore the study of these groups has implications to 4-dimensional symplectic topology. There are also converse results; there are 4-dimensional topological theorems that have implications to mapping class group theory. For compact 4-manifolds with nonempty boundary a very similar correspondence can be set up, provided the manifolds admit Stein structures and we consider mapping class groups of surfaces with nonempty boundary.

The aim of the present survey is to review basic definitions, constructions and theorems of smooth 4-manifold topology (such as Lefschetz fibrations, Seiberg-Witten invariants, Stein structures, and Taubes' theorems) relevant to the study of mapping class groups, and describe some simple proofs of statements connecting the two branches. The proofs are typically only samples; we avoid technically challenging arguments and provid those steps that show how a 4-dimensional result can be applied to studying mapping classes. 
Acknowledgements This paper is the written version of the author's expository lecture at the conference Interactions between low-dimensional topology and mapping class groups held at the Max-Planck-Institute in July 2013. We would like to thank the organizers for the productive conference, and an anonymous referee for many helpful comments and suggestions. The author was supported by the Lendület project ADT of the Hungarian Academy of Sciences and by ERC Grant LTDBud. The present work is part of the authors' activities within CAST, a Research Network Program of the European Science Foundation.

\section{Symplectic manifolds and Taubes' theorems}

Suppose that $X^{4}$ is a smooth, closed, oriented 4 -manifold. A 2 -form $\omega \in \Omega^{2}(X)$ is a symplectic form if:

- $\omega$ is closed, that is, $d \omega=0$.

- $\omega$ is nondegenerate, that is, for any nonzero tangent vector $v \in T_{x} X \quad(x \in X)$ there is another vector $w \in T_{X} X$ with the property that $\omega(v, w) \neq 0$. Equivalently, $\omega \wedge \omega$ is a volume form on $X$. Note that $\omega \wedge \omega$ provides an orientation for $X$; we always assume that this orientation coincides with the given orientation of $X$.

The 2-form $\omega$ defines a symplectic structure (in the linear algebraic sense, that is, a skew symmetric 2-form) on every fiber $T_{x} X$. It is not hard to see that a vector space equipped with a symplectic form admits a complex structure, that is, an endomorphism $J$ with $J^{2}=-\mathrm{Id}$; in fact $J$ can be chosen to be compatible with the given symplectic structure, meaning that $\omega(v, J v)>0$ once $v \neq 0$ and $\omega(J v, J w)=\omega(v, w)$. Once again, linear algebra shows that the space of such endomorphisms is contractible. In particular, by viewing $\omega$ as an element of $\Omega^{2}(X)$ (and hence giving a symplectic structure on every tangent space), the space of all fiberwise compatible almost complex structures form a bundle with contractible fiber, hence admits a section, providing an almost complex structure (still denoted by $J$ ) on $X$ : a bundle endomorphism $J: T X \rightarrow T X$ with $J^{2}=-\operatorname{Id}_{T X}$. Furthermore, the contractibility of the fiber implies that two such sections are homotopic.

Note that $J$ turns $T X$ into a complex bundle, hence $(T X, J)$ admits Chern classes, and since homotopic $J$ 's give rise to equal Chern classes for a closed symplectic 4-manifold $(X, \omega)$, we get two elements $c_{1}(X, \omega) \in H^{2}(X ; \mathbb{Z})$ and $c_{2}(X, \omega) \in H^{4}(X ; \mathbb{Z})$, which are invariants of the symplectic structure. A simple argument shows that $c_{2}(X, \omega)$ is the Euler class of $X$, while for $c_{1}(X, \omega)$ we have:

- The mod 2 reduction of $c_{1}(X, \omega)$ is equal to the second Stiefel-Whitney class $w_{2}(X) \in H^{2}\left(X ; \mathbb{Z}_{2}\right)$. 
- The evaluation of $c_{1}^{2}(X, \omega)$ on the fundamental class $[X]$ is equal to $3 \sigma(X)+$ $2 \chi(X)$, where $\sigma(X)$ is the signature of the intersection pairing on the second (co)homology of $X$ and $\chi(X)$ is the Euler characteristic of $X$.

Remark 2.1 Indeed, by a classical result of Hirzebruch and Hopf [24], the existence of a class $h \in H^{2}(X ; \mathbb{Z})$ satisfying the above two properties (ie having the required mod 2 reduction and square) implies the existence of an almost complex structure $J$ on $X$. This condition, however, is far from being sufficient for the existence of a symplectic structure.

Symplectic 4-manifolds admit various special properties. Most notably, they exhibit features reminiscent in some sense to complex surfaces. These properties can be most conveniently described using Seiberg-Witten invariants. Before turning to the description of these properties, we very quickly recall (at least the formal properties of) the Seiberg-Witten invariants.

Suppose that $X$ is an oriented, closed, smooth 4-manifold with $b_{2}^{+}(X)>1$. (Recall that $b_{2}^{+}(X)$ is the dimension of the maximal positive-definite subspace of $H^{2}(X ; \mathbb{R})$ for the cup product; $b_{2}^{-}(X)$ is defined similarly by considering the maximal dimension of negative-definite subspaces, and the signature $\sigma(X)$ is by definition equal to $b_{2}^{+}(X)-$ $b_{2}^{-}(X)$.) If we fix an orientation of the space $H_{+}^{2}(X ; \mathbb{R}) \oplus H^{1}(X ; \mathbb{R})$ (ie we fix a homology orientation for $X$ ), then the Seiberg-Witten invariant $\mathrm{SW}_{X}$ of $X$ is a map

$$
\mathrm{SW}_{X}: H^{2}(X ; \mathbb{Z}) \longrightarrow \mathbb{Z}
$$

which has finite support, and for a diffeomorphism $f: X_{1} \rightarrow X_{2}$ and $\alpha \in H^{2}\left(X_{2} ; \mathbb{Z}\right)$ it satisfies

$$
\operatorname{SW}_{X_{2}}(\alpha)= \pm \operatorname{SW}_{X_{1}}\left(f^{*}(\alpha)\right) .
$$

(The sign originates from the possibly different choices of homology orientations.) Indeed, the definition can be refined to get a function which is defined on the set of $\operatorname{spin}^{c}$ structures rather than on the second cohomology. Since every $\operatorname{spin}^{c}$ structure gives rise to an element of $H^{2}(X ; \mathbb{Z})$ by taking its first Chern class, the above definition follows from the refined one by summing the refined invariant for all (the finitely many) $\operatorname{spin}^{c}$ structures with a given first Chern class.

For a fixed Riemannian metric $g$ and a $\operatorname{spin}^{c}$ structure $\mathfrak{s}$ on $X$, the Seiberg-Witten invariant counts the number of solutions of a system of partial differential equations associated to the metric and $\mathfrak{s}$ on the 4-manifold $X$ (using the twisted Dirac operator construction and the Hodge star operator). The condition $b_{2}^{+}(X)>1$ ensures that the resulting number will be independent of the chosen metric (and of the appropriate 
perturbation of the equation). For details of the construction of the invariant, see [37; $38 ; 46]$.

A cohomology class $K$ with nonzero Seiberg-Witten value $\mathrm{SW}_{X}(K)$ is called a (Seiberg-Witten) basic class. The relation between basic classes and the geometry of the underlying 4-manifold is most transparent through the adjunction inequality:

Theorem 2.2 (Kronheimer and Mrowka [29; 30]) Suppose that $\Sigma \subset X$ is a smoothly embedded, oriented, closed surface of genus $g(\Sigma)>0$ in the oriented 4-manifold $X$, representing the homology class $[\Sigma] \in H_{2}(X ; \mathbb{Z})$ with the additional property that the self-intersection $[\Sigma]^{2}$ of $\Sigma$ is nonnegative. Then for any basic class $K \in H^{2}(X ; \mathbb{Z})$ we have the adjunction inequality

$$
2 g(\Sigma)-2 \geq[\Sigma]^{2}+|\langle K,[\Sigma]\rangle|
$$

In some sense the above inequality is the smooth version of the adjunction equality from complex geometry, which we state in Equation (2-1) below. Suppose that $C \subset X$ is an embedded, oriented, closed surface of genus $g(C)$ in the symplectic 4-manifold $(X, \omega)$. Fix an almost complex structure $J$ compatible with $\omega$ and assume that $C$ is a complex submanifold for $J$. (This simply means that $T C \subset T X$ is $J$-invariant.) By splitting $T X$ along $C$ as the sum $T C \oplus \nu C$ of complex line bundles, we get the adjunction equality

$$
2 g(C)-2=[C]^{2}-\left\langle c_{1}(X, \omega),[C]\right\rangle
$$

The formula of Theorem 2.2 generalizes Equation (2-1) in the sense that, as the next result shows, $\pm c_{1}(X, \omega)$ is always a basic class:

Theorem 2.3 (Taubes [44]) Suppose that $(X, \omega)$ is a closed symplectic 4-manifold with Chern class $c_{1}(X, \omega) \in H^{2}(X ; \mathbb{Z})$. Then

$$
\mathrm{SW}_{X}\left( \pm c_{1}(X, \omega)\right)= \pm 1
$$

In particular, the first Chern class of a symplectic manifold is always a basic class.

Indeed, Taubes proved a correspondence between Seiberg-Witten basic classes and homology classes which admit $J$-holomorphic representatives. (More precisely, following ideas of Gromov, he introduced a count of $J$-holomorphic representatives of a given homology class and identified this count with the Seiberg-Witten value on an associated cohomology class.) The precise statement of this result is somewhat technical, hence here we will restrict ourselves to spelling out a consequence which will be relevant later. 
Theorem 2.4 (Taubes [45]) Suppose that $(X, \omega)$ is a symplectic 4-manifold with $b_{2}^{+}(X)>1$ and $J$ is a generic compatible almost complex structure. Suppose that $K \in H^{2}(X ; \mathbb{Z})$ is a Seiberg-Witten basic class, that is, $\operatorname{SW}_{X}(K) \neq 0$. Then there is a (not necessarily connected) complex submanifold $C \subset(X, J)$ such that the Poincaré dual $\mathrm{PD}[C]$ of the homology class $[C]$ represented by $C$ is equal to $\frac{1}{2}\left(K-c_{1}(X, \omega)\right)$. Since $\operatorname{SW}_{X}\left(-c_{1}(X, \omega)\right)= \pm 1$, this implies that there is a (not necessarily connected) complex submanifold $C \subset(X, J)$ satisfying

$$
\operatorname{PD}[C]=-c_{1}(X, \omega) .
$$

This deep result then provides a simple consequence which will be applied in studying properties of mapping class groups:

Corollary 2.5 Suppose that $(X, \omega)$ is a symplectic 4-manifold with $b_{2}^{+}(X)>1$ which contains no embedded sphere of self-intersection -1 . Then $c_{1}^{2}(X, \omega) \geq 0$.

Proof Let $J$ be a generic compatible almost complex structure on $(X, \omega)$ and suppose that $C_{i}$ is a component of the $J$-holomorphic submanifold $C$ representing $-c_{1}(X, \omega)$. Then $-\left\langle c_{1}(X, \omega),\left[C_{i}\right]\right\rangle=\left[C_{i}\right]^{2}$, hence the adjunction equality of (2-1) provides $g\left(C_{i}\right)-$ $1=\left[C_{i}\right]^{2}$. Now either $g\left(C_{i}\right)$ is zero (hence $C_{i}$ is an embedded sphere) and then $\left[C_{i}\right]^{2}=-1$, or $\left[C_{i}\right]^{2} \geq 0$. By assumption $X$ contains no sphere of square -1 , hence $\left[C_{i}\right]^{2} \geq 0$ holds for every component. Summing these contributions for all (disjoint) components we get the claimed statement.

Remark 2.6 In fact, using Taubes' correspondence it can be shown that $(X, \omega)$ with $b_{2}^{+}(X)>1$ contains an embedded -1 -sphere exactly if it contains a $J$-holomorphic -1 -sphere for any compatible almost complex structure $J$.

We will pay special attention to characteristic numbers of symplectic 4-manifolds; the following conjecture of Gompf is along those lines. It is concerned with the sign of the Euler characteristic $\chi$ of a symplectic 4-manifold $X$ : we expect $\chi(X)$ to be nonnegative, with the exception of some well-known cases. More precisely:

Conjecture 2.7 Suppose that $(X, \omega)$ is a symplectic 4 -manifold. Then either $X$ is the blow-up of an $S^{2}$-bundle over a genus- $g$ surface (in which case the genus $g$ and the number of blow-ups determine the Euler characteristic $\chi(X))$, or $X$ has nonnegative Euler characteristic $\chi(X)$. 


\section{Lefschetz fibrations and Lefschetz pencils}

Lefschetz fibrations were originally introduced to study topological properties of projective varieties. Indeed, suppose that $X \subset \mathbb{C P}^{n}$ is a complex surface. Let $A$ (an axis) be given as the transverse intersection of two hyperplanes; in particular, $A$ is diffeomorphic to $\mathbb{C P}^{n-2}$. Then $A$ defines a map $f: X-A \rightarrow \mathbb{C P}^{1}$ as follows: parametrize the hyperplanes of $\mathbb{C P}^{n}$ containing $A$ by $\mathbb{C P}^{1}$, and associate to $x \in X-A$ the parameter $t \in \mathbb{C P}^{1}$ of the hyperplanes $H_{t}$ containing both $A$ and $x$. (Notice that this map is undefined at the points of $A$.) Blow up $X$ along $X \cap A$, resulting in a variety $\widetilde{X}_{A}$. The above map $f$ obviously extends to $\tilde{f}: \tilde{X}_{A} \rightarrow \mathbb{C P}^{1}$, and for a generic choice of $A$ it can be shown that $d \tilde{f}_{p}$ is onto with finitely many exceptions, around which there are appropriate complex charts on which $\tilde{f}=z_{1}^{2}+z_{2}^{2}$.

Notice that since $X$ is of complex dimension 2, for a generic choice of $A$, the intersection $X \cap A$ is a finite set of points. In smooth terminology the blow-up simply means the connected sum with $\overline{\mathbb{C P}}^{2}$, the complex projective plane with the orientation opposite to the one induced by the complex structure. The above construction motivates the following definition:

Definition 3.1 Suppose that $X$ is a smooth, closed, oriented $4-$ manifold, and $\Sigma$ is a smooth, closed, oriented 2-manifold. The smooth map $f: X \rightarrow \Sigma$ is a Lefschetz fibration (with base $\Sigma$ ) if $d f_{p}$ is onto with finitely many exceptions $C=\left\{p_{1}, \ldots, p_{m}\right\}$ (the set of critical points of $f$ ), for which points there are orientation-preserving complex charts $U_{i} \subset X$ and $V_{i} \subset \Sigma\left(p_{i} \in U_{i}\right.$ and $\left.f\left(p_{i}\right) \in V_{i}\right)$ with the property that $\left.f\right|_{U_{i}}=z_{1}^{2}+z_{2}^{2}$ (as a map $U_{i} \rightarrow V_{i}$ ). Informally, we require $f$ to be a complex Morse function. If for some $t \in \Sigma$ the intersection $f^{-1}(t) \cap C$ is nonempty, the fiber $f^{-1}(t)$ of $f$ is called a singular fiber.

With the above terminology in place, in our starting construction we can say that for a generic choice of $A$, an appropriate blow-up $\tilde{X}_{A}$ of a complex projective surface $X$ admits a Lefschetz fibration with base $\mathbb{C P}^{1}$.

Recall that in our earlier arguments spheres of square -1 played a crucial role. Indeed, a manifold containing no -1 -sphere is called minimal. A version of this concept is adapted to Lefschetz fibrations: a Lefschetz fibration $f: X \rightarrow \Sigma$ is relatively minimal if there is no -1 -sphere in $X$ which is contained in a fiber of $f$. This condition ensures that we cannot contract a -1 -sphere in $X$ in a way that the fibration structure persists to the quotient.

The definition of a Lefschetz fibration admits a straightforward modification to include the map we found in the complex projective setting before the blow-up: 
Definition 3.2 Suppose that $X$ is a smooth, closed, oriented 4-manifold. A Lefschetz pencil on $X$ consists of a nonempty finite set $B \subset X$ and a map $f: X-B \rightarrow \mathbb{C P}^{1}$ with the following two properties:

- There is an orientation-preserving complex chart $U_{b}$ for every element $b \in B$ on which $f$ restricts to the projectivization map $\mathbb{C}^{2}-\{0\} \rightarrow \mathbb{C P}^{1}$.

- At each critical point $f$ behaves as it would in a Lefschetz fibration, that is, there are orientation-preserving complex charts $U_{i} \subset X$ and $V_{i} \subset \mathbb{C P}^{1}$ with $\left.f\right|_{U_{i}}=z_{1}^{2}+z_{2}^{2}$.

By blowing up the points of $B \subset X$ we get a Lefschetz fibration $\tilde{X} \rightarrow \mathbb{C P}^{1}$ on the blown-up manifold $\tilde{X}=X \#_{|B|} \overline{\mathbb{C P}}^{2}$ (with base $\mathbb{C P}^{1}$ ).

It is a natural question to examine which 4-manifolds admit a Lefschetz pencil structure. Surprisingly, the (purely topological) property of carrying a Lefschetz fibration is equivalent to the (more analytic) property of admitting a symplectic structure. More formally:

Theorem 3.3 (Donaldson [13]) Suppose that $X$ is a closed, symplectic 4-manifold. Then $X$ admits a Lefschetz pencil. Consequently, for every closed, symplectic 4manifold there is an integer $n$ such that the $n$-fold blow-up $X \# \#_{1}^{n} \overline{\mathbb{C P}}^{2}$ admits a Lefschetz fibration.

Indeed, the converse of the above result of Donaldson also holds:

Theorem 3.4 (Gompf $[19 ; 20]$ ) Suppose that the closed, oriented 4-manifold $X$ admits a Lefschetz pencil with nonempty base $B$, or a Lefschetz fibration with the property that the fiber is nontrivial in homology (when taken with coefficients in $\mathbb{R}$ ). Then $X$ admits a symplectic structure.

In fact, if the genus of the fiber of a Lefschetz fibration on the closed 4-manifold $X$ is not equal to one, then the homology class $[F]$ represented by the generic fiber $f$ is nontrivial in real homology. Indeed, using the fibration one can build an almost complex structure $J$ with the property that the generic fiber is a $J$-holomorphic submanifold, hence by the adjunction equality we get that the value of $c_{1}(X, J)$ on the homology class $[F]$ of the fiber is equal to $2 g(F)-2$ (since $[F]^{2}=0$ for a fiber). If $g(F) \neq 1$, this evaluation shows that $[F] \neq 0$ in $H_{2}(X ; \mathbb{R})$.

For torus fibrations, however, the total space $X$ might not be symplectic: the product of the Hopf fibration $S^{3} \rightarrow S^{2}$ with $S^{1}$ gives a nontrivial torus bundle (a Lefschetz fibration with no singular fibers) on $S^{3} \times S^{1}$, but since $H^{2}\left(S^{3} \times S^{1}\right)=0$, we do not 
have a closed 2-form (representing a second cohomology class) which is nondegenerate. Notice furthermore that if the Lefschetz fibration $\tilde{X} \rightarrow \mathbb{C P}^{1}$ comes from blowing up a Lefschetz pencil, then the fiber is nontrivial in homology: the exceptional sphere in any of the blow-ups provides a section, hence a submanifold intersecting the fiber in a single (transverse) point. In fact, on a fibration the symplectic structure can be constructed in such a way that any preassigned finite set of disjoint sections will be symplectic, hence by blowing them back down, the pencil case in Theorem 3.4 follows easily from the fibration case. For further details, see [21, Section 10.2].

We will pay special attention to sections of Lefschetz fibrations. If $f: X \rightarrow \Sigma$ is a given Lefschetz fibration, then a section of $f$ is a map $\sigma: \Sigma \rightarrow X$ with the property that $f \circ \sigma=\mathrm{id}_{\Sigma}$. Sometimes we will confuse the section (a map) with its image $\sigma(\Sigma)$ (a subset of $X$ ), and even with the homology class it represents, and consider the self-intersection (or square) of a section, referring to the corresponding number of the homology class $[\sigma(\Sigma)]$.

\section{Lefschetz fibrations and mapping class groups}

Suppose that $f: X \rightarrow \Sigma$ is a Lefschetz fibration. It is not hard to see that, by possibly slightly perturbing $f$, one can assume that $f$ is injective on the set $C_{f}$ of critical points of $f$. Suppose now that $x \in \Sigma$ is a critical value, that is, $f^{-1}(x)$ is a singular fiber. By the above assumption it follows that $f^{-1}(x)$ contains a unique critical point. The topology of the neighborhood of the fiber $f^{-1}(x)$ can be given as follows: Pick a regular value $y$ near $x$ and an embedded arc from $y$ to $x$. Consider a simple closed curve inside the tubular neighborhood of the chosen arc which passes through $y$ and encircles $x$ (and does not pass through any critical value). Restricting the Lefschetz fibration to this loop we get a surface bundle over $S^{1}$ (since the loop avoids all the critical values). Such a fibration can be described by its monodromy: considering it over $[0,1]$ we get a bundle with a canonical trivialization, and the map (defined up to conjugation and isotopy) identifying the fibers over 0 and 1 is the monodromy of the fibration over $S^{1}$. In case the surface bundle is given by the procedure above (restricting a Lefschetz fibration to a loop encircling a unique Lefschetz critical point), the monodromy can be shown to be a right-handed Dehn twist along a simple closed curve $c=c_{x}$. Indeed, the neighborhood of a singular fiber can be given by attaching a 4-dimensional 2-handle to the neighborhood of the generic fiber along $c$ (with framing $(-1)$ relative to the framing the curve inherits from the fiber). This observation quickly leads to a handlebody description of a Lefschetz fibration. This approach is very useful in many problems, but since we will not exploit it in this survey, we do not 
go into the description of the details of the handlebody picture. For further discussion on the handlebody description, see [21].

The simple closed curve $c$ (specified by the Dehn twist up to isotopy) is called the vanishing cycle corresponding to the singular fiber. The terminology is justified by the fact that this circle bounds a disk in $X$, hence represents the zero cycle in the 4-manifold. The disk (which can also be interpreted as the core of the 2-handle we mentioned above) is called the Lefschetz thimble associated to the singular fiber.

The above idea of considering the monodromy can be extended to any based loop avoiding critical values. The monodromy representation of a Lefschetz fibration $f: X \rightarrow \Sigma$ is the map

$$
m_{f}: \pi_{1}\left(\Sigma-f\left(C_{f}\right)\right) \longrightarrow \Gamma_{g}
$$

which associates to a loop in $\Sigma-f\left(C_{f}\right)$ (hence to a loop avoiding the critical values) the monodromy of the surface bundle over that loop. (Strictly speaking, since loops concatenate from left to right, while diffeomorphisms act on the left, this map is an antihomomorphism, which can be turned to a homomorphism by composing it with the inversion map $g \mapsto g^{-1}$. We will always assume that we have applied this composition.) The fundamental group $\pi_{1}\left(\Sigma-f\left(C_{f}\right)\right)$ admits a simple presentation

$$
\left\langle z_{1}, \ldots, z_{n}, a_{1}, b_{1}, \ldots, a_{g}, b_{g} \mid \prod_{i=1}^{n} z_{i} \prod_{j=1}^{g}\left[a_{j}, b_{j}\right]=1\right\rangle,
$$

where $z_{i}$ can be represented by small loops around the critical values, while $\left\{a_{1}, b_{1}\right.$, $\left.\ldots, a_{g}, b_{g}\right\}$ can be chosen to be a usual generating set of $\pi_{1}(\Sigma)$.

Since the homomorphism $m_{f}$ maps $z_{i}$ to a right-handed Dehn twist $t_{i}$, the map $m_{f}$ can be determined by the factorization

$$
\prod_{i=1}^{n} t_{i} \prod_{j=1}^{g}\left[\alpha_{j}, \beta_{j}\right]=1 \in \Gamma_{g}
$$

in the mapping class group $\Gamma_{g}$, where $t_{i}=m_{f}\left(z_{i}\right)$ is a right-handed Dehn twist, and $\alpha_{j}=m_{f}\left(a_{j}\right)$ and $\beta_{j}=m_{f}\left(b_{j}\right)$ are mapping classes with no particular property.

This approach can capture further structures. Indeed, consider the mapping class group $\Gamma_{g, 1}$ of those mapping classes which fix a preassigned point $p \in \Sigma$. There is an obvious map $\Gamma_{g, 1} \rightarrow \Gamma_{g}$, since a mapping class fixing the point $p$ naturally gives rise to a mapping class. By the Birman exact sequence (see [18, Theorem 4.6]), the kernel of this map can be identified with $\pi_{1}(\Sigma)$, hence there are various choices for an element in $\Gamma_{g}$ to be lifted to $\Gamma_{g, 1}$. For example, for a Dehn twist along a simple closed curve $C$ the lift depends on the isotopy class of $C$ relative to $p$. 
It is easy to see that if the factorization $\prod_{i=1}^{n} t_{i} \prod_{j=1}^{g}\left[\alpha_{j}, \beta_{j}\right]=1 \in \Gamma_{g}$ admits a lift $\prod_{i=1}^{n} \widetilde{t}_{i} \prod_{j=1}^{g}\left[\widetilde{\alpha}_{j}, \widetilde{\beta}_{j}\right]=1 \in \Gamma_{g, 1}$, then the corresponding Lefschetz fibration admits a section. Indeed, the fixed point $p$ defines a submanifold in $X$ which intersects each fiber in a single point, hence provides a section of the Lefschetz fibration.

A further group $\Gamma_{g}^{1}$ can be defined by considering mapping classes of maps which fix a point $p$ and a tangent vector $v$ at $p$. (Alternatively, we can consider the surface $\Sigma-v(p)$ with boundary, where $v(p)$ is a small open neighborhood of $p$, and take mapping classes which fix the boundary pointwise.) Obviously there is a natural map $\Gamma_{g}^{1} \rightarrow \Gamma_{g, 1}$, and the kernel is isomorphic to $\mathbb{Z}$, consisting of powers of the Dehn twist $\delta$ along a simple closed curve parallel to the boundary. Now each element of $\Gamma_{g, 1}$ admits a lift to $\Gamma_{g}^{1}$, and hence $\prod_{i=1}^{n} \widetilde{t}_{i} \prod_{j=1}^{g}\left[\widetilde{\alpha}_{j}, \widetilde{\beta}_{j}\right]$ lifts to

$$
\prod_{i=1}^{n} \bar{t}_{i} \prod_{j=1}^{g}\left[\bar{\alpha}_{j}, \bar{\beta}_{j}\right] \text {. }
$$

The expression is a relation in $\Gamma_{g, 1}$, therefore it is in the kernel of the map $\Gamma_{g}^{1} \rightarrow \Gamma_{g, 1}$. Consequently, the product of Equation (4-2) is equal to $\delta^{n}$ for some $n \in \mathbb{Z}$. The geometric significance of this integer is easy to see: $-n$ is the self-intersection of the section given by the relation (viewed the section as an oriented surface in the 4-manifold $X)$. Indeed, by fixing a point $q \in \partial v(p)$, we get a push-off of the section intersecting it in $n$ points; a local calculation shows that all intersection points come with the same sign, and in a model case we can identify the sign of the points to be equal to $(-1)$, giving the claimed self-intersection.

The ideas above extend to capturing $k$ disjoint sections by lifting the monodromy from $\Gamma_{g}$ to $\Gamma_{g, k}$. The self-intersections can be identified by taking the factorizations in $\Gamma_{g}^{k}$ : the result will be equal to $\delta_{1}^{n_{1}} \cdots \delta_{k}^{n_{k}}$, where $\delta_{i}$ is the right-handed Dehn twist along a curve parallel to the $i^{\text {th }}$ boundary component, and the exponents $n_{i}$ are the negatives of the self-intersections of the sections.

Recall that a Lefschetz pencil can be blown up to a Lefschetz fibration, and by keeping track of the exceptional spheres, this procedure can be reversed. On the other hand, the $k$ exceptional spheres are all section of the resulting fibration with self-intersection -1 , hence - according to what was said above - the monodromy representation can be considered in $\Gamma_{g}^{k}$, factoring $\prod_{i=1}^{k} \delta_{i}$ (where the $\delta_{i}$ are the right-handed Dehn twists corresponding to the fixed points originating from the sections). This approach therefore provides a mapping class group-theoretic description of a Lefschetz pencil, hence of a symplectic 4-manifold.

Note that the existence of a lift of the relation from $\Gamma_{g}$ to $\Gamma_{g, 1}$ is far from trivial. Indeed, since there are surface bundles over surfaces which do not admit a section (see 
Hillman [23]), we cannot expect that any factorization of 1 in $\Gamma_{g}$ lifts to a factorization of 1 in $\Gamma_{g, 1}$. There is, however, no example of a Lefschetz fibration over $S^{2}$ without a section. This fact leads us to the following problem.

Problem 4.1 Prove (or disprove) that any nontrivial Lefschetz fibration over $S^{2}$ (ie $f: X \rightarrow S^{2}$ with at least one singular fiber with homotopically nontrivial vanishing cycle) admits a section. (Nontrivial Lefschetz fibrations over $S^{2}$ with fiber genus 1 do admit sections, and some partial results along these lines can be derived from work of Siebert and Tian [39] for $g=2$.)

Remark 4.2 The positive resolution of this problem would be of utmost importance in the handlebody description of a Lefschetz fibration: the complement of a generic fiber admits a straightforward description in terms of the vanishing cycles, but the addition of the 2-handle of the neighborhood of the generic fiber admits a simple description only when the fibration admits a section.

\section{Singular fibers in fibrations over $S^{2}$}

Let us now concentrate on fibrations over $S^{2}$. It is a natural question to determine the minimal number of singular fibers a nontrivial Lefschetz fibration of fiber genus $g$ should have. In mapping class group terms, it asks for the minimal length of a nontrivial factorization of the identity in $\Gamma_{g}$ into the product of right-handed Dehn twists.

Relying on Taubes' result from Section 2, it is not hard to come up with a linear lower bound in the fiber genus $g$, which we explain below. Consider a nontrivial Lefschetz fibration $f: X \rightarrow S^{2}$ with $k>0$ singular fibers, and with corresponding monodromy factorization $\prod_{i=1}^{k} t_{i}=1$.

In the following arguments we will need a simple construction of Lefschetz fibrations.

Definition 5.1 Suppose that $f_{i}: X_{i} \rightarrow \Sigma_{i}(i=1,2)$ are two genus- $g$ Lefschetz fibrations. Fix a regular fiber in each and delete a tubular neighborhood of these fibers. An identification of the chosen regular fibers in the two fibrations extends to an identification of their neighborhoods, and composing this identification with the map we get from the complex conjugation on the base disk we get a fiber-preserving, orientation-reversing diffeomorphism of the boundaries. Gluing the two fibrations over $\Sigma_{i}-D^{2}$ together with this diffeomorphism we get the fiber sum $X_{1} \#_{f} X_{2}$ of $X_{1}$ and $X_{2}$, which is a Lefschetz fibration over the surface $\Sigma_{1} \# \Sigma_{2}$. 
Let us take the Lefschetz fibration $X \#_{f} X \rightarrow S^{2}$, the fiber sum of $X$ with itself. We can also describe this fibration through its monodromy factorization; it is

$$
\left(\prod_{i=1}^{k} t_{i}\right) \cdot\left(\prod_{i=1}^{k} t_{i}\right)=\left(\prod_{i=1}^{k} t_{i}\right)^{2}=1 .
$$

The following two facts can be verified:

Lemma 5.2 [40] Suppose that $X \rightarrow S^{2}$ is a nontrivial, relatively minimal Lefschetz fibration. Then $X \#_{f} X$ is a minimal symplectic 4-manifold with $b_{2}^{+}\left(X \#_{f} X\right)>1$.

Remark 5.3 The fact that $b_{2}^{+}\left(X \#_{f} X\right)>1$ follows from the nontriviality of the fibration: Recall that a vanishing cycle bounds a disk with (relative) framing -1 (which was called the Lefschetz thimble corresponding to the critical point). Since vanishing cycles in $X \#_{f} X$ appear in pairs, the union of two thimbles corresponding to homologically nontrivial vanishing cycles provides a $-2-$ sphere, with a dual torus (hence a hyperbolic pair) with the fiber in the complement. This dual torus can be visualized as follows: take the product of the circle dual to the vanishing cycle with the circle in the base we get when in the fiber sum operation we delete the tubular neighborhood of a regular fiber (and hence a $D^{2}$ from the base). The minimality of the fibration requires a slightly more complicated argument; see [40].

The above lemma (together with Corollary 2.5 of Taubes' result) then easily implies:

Proposition 5.4 [40] Suppose that $X \rightarrow S^{2}$ is a nontrivial, relatively minimal Lefschetz fibration with $k$ singular fibers. Then

$$
\frac{4}{5}(g-1) \leq k .
$$

Proof Let us consider $X \#_{f} X$. Since by Lemma 5.2 it is a minimal symplectic 4-manifold with $b_{2}^{+}\left(X \#_{f} X\right)>1$, by Corollary 2.5 we have that $c_{1}^{2}\left(X \#_{f} X\right) \geq 0$. Recall that $c_{1}^{2}=3 \sigma+2 \chi$ and $\sigma$ is simply additive for fiber sums, while $\chi$ satisfies the slightly more complicated formula

$$
\chi\left(X \#_{f} Y\right)=\chi(X)+\chi(Y)+4 g-4
$$

for the fiber sum $X \#_{f} Y$ of the genus- $g$ Lefschetz fibrations $X$ and $Y$. Then the inequality $c_{1}^{2}\left(X \#_{f} X\right) \geq 0$ implies that

$$
4-4 g \leq c_{1}^{2}(X)=3 \sigma(X)+2 \chi(X) \leq 3 k+2(4-4 g+k),
$$

where we applied the bound $\sigma(X) \leq k$ for the signature and the equality $\chi(X)=$ $4-4 g+k$ for the Euler characteristic. This last equality follows from the fact that 
deleting two regular fibers from $X$ (with Euler characteristic $2-2 g$ each) we get a cobordism involving $k$ 2-handles, while the inequality $\sigma(X) \leq k$ is a consequence of $\sigma \leq b_{2} \leq k$ (the second following from the above presentation of $X-\{$ two fibers $\}$ as a union of 2-handles again). The resulting inequality (5-1) implies the claim of the proposition at once.

The best-known bound for the number of singular fibers is due to Braungardt and Kotschick [7]: the number $k$ of singular fibers in a nontrivial Lefschetz fibration over $S^{2}$ with the extra property that the map is injective on the critical points is

$$
k \geq \frac{1}{5}(8 g-3) .
$$

This value is rather close to the value of $k$ realized by the following example (the smallest $k$ known to be realized by a fibration for $g \geq 15$ ):

Example 5.5 Suppose that $g=2 n+1$ is odd, and consider the product complex surface $S^{2} \times \Sigma_{n}$ of $S^{2}=\mathbb{C P}^{1}$ and the genus- $n$ surface $\Sigma_{n}$. Let $C$ denote the singular complex curve in $S^{2} \times \Sigma_{n}$ given by two copies of $\{\mathrm{pt}\} \times \Sigma_{n}$ (for two different points) and four copies of $S^{2} \times\{\mathrm{pt}\}$ (again, for four distinct points). The double branched cover of the complex surface $S^{2} \times \Sigma_{n}$ along $C$ gives a (singular) complex surface, which (after desingularization) gives rise to the complex surface $X$. Indeed, we can first smooth the transverse double points of $C$ to get a smooth curve in $S^{2} \times \Sigma_{n}$ and take the double branched cover afterwards; the resulting smooth manifold will be diffeomorphic to the desingularized surface $X$. A further equivalent way of seeing this construction is to blow up the singular points of $C$ and then take the double branched cover along the proper transform (which is now a smooth curve in the blow-up).

Notice that $X$ comes with two fibration maps: the composition of the resolution of singularities $X \rightarrow \bar{X}$ with the branched cover $\bar{X} \rightarrow S^{2} \times \Sigma_{n}$ can be further composed with the two projections. These maps are holomorphic, and can be perturbed to Lefschetz fibrations. Indeed, the map to $\Sigma_{n}$ is a Lefschetz fibration with fibers given by the double branched cover of $S^{2}$ branched in two points. Therefore it is a ruling on $X$, and it is not hard to show that $X$ is diffeomorphic to $S^{2} \times \Sigma_{n} \# 8 \overline{\mathbb{C P}}^{2}$. The other projection (to $S^{2}$ ) then equips $X$ with a Lefschetz fibration, where the fiber is the double branched cover of $\Sigma_{n}$, branched in four points. This is a genus- $g$ surface (remember, $g=2 n+1$ ), and a simple calculation with the Euler characteristic (based on the formula $\chi(X)=4-4 g+k$ for the number $k$ of singular fibers) shows that the perturbed Lefschetz fibration $X \rightarrow S^{2}$ has $2 g+10$ singular fibers. Indeed, with a little extra effort one can see that the vanishing cycles corresponding to the singular fibers are homologically essential. (A similar construction adapts if $g$ is even, providing a fibration with $2 g+4$ singular fibers; see [42].) 
In conclusion, there are examples of nontrivial fibrations with $2 g+10$ (for odd $g$ ) and $2 g+4$ (for even $g$ ) singular fibers, and we know that any fibration must have at least $\frac{1}{5}(8 g-3)$ singular fibers.

Problem 5.6 Determine the exact value of the minimal number of singular fibers a nontrivial, relatively minimal Lefschetz fibration over $S^{2}$ (with $\left.f\right|_{C_{f}}$ injective) must have. (The answer is classical for $g=1$ : a genus-1 Lefschetz fibration over $S^{2}$ has $12 q(q \in \mathbb{N})$ singular fibers, hence the minimal value is 12 in this case.)

Remark 5.7 The same question over higher-genus surfaces has been addressed and answered by Korkmaz and Ozbagci [27]: if the base genus is at least two and the fiber genus is at least three, there is always a nontrivial, relatively minimal fibration with a single singular fiber (corresponding to the fact that for $g \geq 3$ a Dehn twist can be written as a product of two commutators).

The linear growth of the number of singular fibers can be used to deduce the following simple result regarding self-intersections of sections:

Proposition 5.8 Suppose that $\sigma$ is a section of the nontrivial Lefschetz fibration $f: X \rightarrow S^{2}$ over the sphere. Then the self-intersection of the submanifold $\sigma\left(S^{2}\right) \subset X$ is negative.

Remark 5.9 By the adjunction formula it is rather clear that if $b_{2}^{+}(X)>1$, then the square of the section (which is an embedded sphere in $X$ ) must be negative. If $b_{2}^{+}(X)=1$, however, $X$ might contain spheres of nonnegative square, and in this case the proposition claims that those cannot be sections of any nontrivial Lefschetz fibration.

Proof of Proposition 5.8 Suppose that the genus- $g$ Lefschetz fibration $f: X \rightarrow S^{2}$ admits a section with nonnegative square. After possibly blowing up, we get a genus- $g$ Lefschetz fibration $f^{\prime}: X^{\prime} \rightarrow S^{2}$ with a section of square 0 . Now taking $S^{2} \times \Sigma_{h}$ (which obviously admits a section of square 0 ) we can "section-sum" them, ie glue the fibers of $f^{\prime}$ and of the trivial fibration along the sections of square 0 , resulting in a Lefschetz fibration of genus $g+h$, with the same number of singular fibers as $f^{\prime}$. Taking $h$ large enough, the resulting fibration will contradict Proposition 5.4.

\section{Commutator length in mapping class groups}

A beautiful application of the correspondence between mapping class groups and Seiberg-Witten theory (furnished by Lefschetz fibrations and symplectic 4-manifolds) 
is given by the following result of Endo and Kotschick [17] (and independently Korkmaz [26]; see also Bestvina and Fujiwara [5] for a subsequent, different proof).

Recall that for $g \geq 3$ the mapping class group is perfect, that is, any element in it can be written as a product of commutators. It is natural to ask whether the number of commutators is bounded (that is, the group is uniformly perfect) or no such bound exists. Let us define the commutator length $c(x)$ of an element $x \in \Gamma_{g}$ as the minimal number of commutators one needs to use to express $x$ as a product of commutators.

Theorem 6.1 (Endo and Kotschick [17], Korkmaz [26]) Suppose that $g \geq 3$ and $t \in \Gamma_{g}$ is a right-handed Dehn twist along a separating (but not null-homotopic) curve. Then the sequence $c\left(t^{n}\right)$ is not bounded. Consequently, the mapping class group $\Gamma_{g}$ (with $g \geq 3$ ) is not uniformly perfect.

Proof Suppose that there is an integer $K \in \mathbb{N}$ with $c\left(t^{n}\right) \leq K$, and consider the expressions

$$
t^{n}=\prod_{i=1}^{K}\left[a_{i}(n), b_{i}(n)\right]
$$

expressing $t^{n}$ as the product of $K$ commutators. (In case $c\left(t^{n}\right)<K$, we add further terms with commuting elements, say $a_{i}(n)=1$.) As in Equation (4-1), the relation of Equation (6-1) gives rise to a genus- $g$ Lefschetz fibration $X_{n} \rightarrow \Sigma_{K}$ over the surface $\Sigma_{K}$ of genus $K$ with $n$ singular fibers. The Euler characteristic of $X_{n}$ is easy to determine: the $n$ critical points corresponding to $t^{n}$ in the expression of Equation (6-1) give $n$, while the rest is a $\Sigma_{g}$-bundle over $\Sigma_{K}-D$, hence we get

$$
\chi\left(X_{n}\right)=n+4(g-1)(K-1) .
$$

For the signature, we again consider the decomposition of $X_{n}$ as the union of a Lefschetz fibration over the disk $D$ with the $n$ singular fibers, and the complement admitting a surface bundle structure over $\Sigma_{K}-D$. The former piece has signature $-n$ : Based on the fact that the chosen vanishing cycle is separating, we explicitly see homology classes generating $H_{2}$. The signature of the surface bundle over $\Sigma_{K}-D$ can be bounded by the second Betti number, which is definitely less than the sum of the Euler characteristic and twice the first Betti number, which can be bounded from above by $(2 g-1)(2 K-1)$. Indeed, the exact value of this bound is not important; the important fact is that there is an upper bound of the signature of the fibration over $\Sigma-D$ independent of $n$. Therefore, for $n$ large enough, the square of the first Chern class $c_{1}^{2}\left(X_{n}\right)$ (which is equal to $\left.3 \sigma\left(X_{n}\right)+2 \chi\left(X_{n}\right)\right)$ is roughly $-n$, consequently, for $n$ large enough we have $c_{1}^{2}\left(X_{n}\right)<0$. 
It is not hard to see, however, that we can assume that $b_{2}^{+}\left(X_{n}\right)>1$ (by possibly fiber summing with an appropriate surface bundle), and (just as in Lemma 5.2) we have that $X_{n}$ is minimal. By Corollary 2.5 these two properties provide that $c_{1}^{2}\left(X_{n}\right) \geq 0$, hence the above contradiction shows that our initial assumption on the bounded commutator length of $t^{n}$ is false, verifying the result.

Remark 6.2 The above rough argument shows that the commutator length is unbounded; a more careful analysis using similar arguments provides more precise information about the behavior of the asymptotics of $c\left(t^{n}\right)$; for details, see [17; 26].

In the argument above the simple estimate on the signature of a Lefschetz fibration played an important role. Indeed, the signature of a Lefschetz fibration seems to obey certain constraints. For example, no nontrivial Lefschetz fibration $X \rightarrow S^{2}$ is known which admits nonnegative signature. This leads us to the following problem:

Problem 6.3 Show that a nontrivial Lefschetz fibration $X \rightarrow S^{2}$ has negative signature.

Indeed, this question can be put in a slightly broader context:

Problem 6.4 Show that for any two fixed integers $g, h \in \mathbb{N}$ there is an integer $N(g, h)$ with the property that if $X \rightarrow \Sigma_{h}$ is a genus- $g$ Lefschetz fibration over the surface of genus $h$, then the signature $\sigma(X)$ is at most $N(g, h)$.

It is easy to see that if such an $N(g, 0)$ (or $N(g, 1))$ exists, then it can be chosen to be zero: If there is a Lefschetz fibration $X \rightarrow S^{2}$ with positive signature $\sigma(X)$, then the $n$-fold fiber sum $X \#_{f} \cdots \#_{f} X$ has signature $n \sigma(X)$, which can then grow arbitrarily large, contradicting the existence of $N(g, 0)$. Similarly, a genus- $g$ Lefschetz fibration $X \rightarrow T^{2}$ over the torus $T^{2}$ with positive signature can be pulled back by an $n$-fold (unramified) cover $f: T^{2} \rightarrow T^{2}$, resulting in $X_{n}=f^{*}(X)$ with $\sigma\left(X_{n}\right)=n \sigma(X)$; once again, contradicting the existence of $N(g, 1)$.

\section{Surface bundles over surfaces}

Motivated by the above considerations, we will examine possible signatures of surface bundles (ie Lefschetz fibration without singular fibers) in more details. It is a simple fact that the Euler characteristic is multiplicative, hence for a genus- $g$ surface bundle $X \rightarrow \Sigma_{h}$ we have that $\chi(X)=4(g-1)(h-1)$. The computation of the signature, however, turns out to be a much more subtle question. Multiplying the "signatures" of 
the base $\Sigma_{h}$ and the fiber $\Sigma_{g}$ (both zero), we expect a surface bundle to have vanishing signature. This vanishing of the signature of a surface bundle over a surface indeed holds for special fibrations (as shown by a classical result of Chern, Hirzebruch and Serre [10]). The fact that the vanishing of the signature is not always true for surface bundles over surfaces was shown by Atiyah [2] and (independently) Kodaira [25]. The question has been further studied since then; see $[8 ; 15 ; 16]$. Here we only restrict ourselves to a simple construction providing examples of surface bundles with nonvanishing signature (see also [9]), and then recall some recent developments in determining relations among characteristic numbers of surface bundles.

Consider the genus- $g$ Lefschetz fibration $X \rightarrow S^{2}$ constructed in Example 5.5, which is diffeomorphic to $S^{2} \times \Sigma_{n} \# 8 \overline{\mathbb{C P}}$ (with $g=2 n+1$ ), and hence has $\sigma(X)=-8$. Recall that (since the mapping class group $\Gamma_{g}$ is perfect once $g \geq 3$ ) any right-handed Dehn twist $t$ can be written as a product of commutators. The resulting relation $t \prod_{i=1}^{m}\left[a_{i}, b_{i}\right]=1$ defines a Lefschetz fibration $Y \rightarrow \Sigma_{m}$ with a single singular fiber over the surface of genus $m$. Since for any almost complex manifold the sum $\sigma+\chi$ is divisible by 4 , and $\chi(Y)=1+4(g-1)(m-1)$, we get that $\sigma(Y) \equiv-1(\bmod 4)$. Assume that $t$ is a Dehn twist along a homologically essential simple closed curve, and recall that the monodromy of $X$ of Example 5.5 can be factored to a product of Dehn twists along homologically essential simple closed curves. (This fact is nontrivial, yet not very hard to verify.) Since any two homologically essential simple closed curves on a surface can be mapped into each other by a diffeomorphism of the surface, it is not hard to see that the tubular neighborhoods of such fibers in any two Lefschetz fibrations are (fiber preserving) diffeomorphic. Therefore, we can consider the singular fiber sums of $X$ and $2 g+10$ copies of $Y$ : Suppose that $G_{i} \subset X$ is the tubular neighborhood of the $i^{\text {th }}$ singular fiber, consider

$$
X-\bigcup_{i=1}^{2 g+10} G_{i}
$$

and $2 g+10$ copies of

$Y$ - (the tubular neighborhood of the single singular fiber),

and glue each of the latter to $X-\bigcup_{i=1}^{2 g+10} G_{i}$ along the diffeomorphic boundaries. Since we can glue these manifolds together with fiber-preserving, orientation-reversing diffeomorphisms of the respective boundaries, we can construct a surface bundle $Z \rightarrow \Sigma_{k}$, where $k=m(2 g+10)$ (recall that $m$ is the number of commutators in the factorization of the Dehn twist $t$ ). Since the boundary components are orientationpreserving diffeomorphic, we need to reverse the orientations of the $Y$ so we can glue 
the pieces together. Novikov additivity for the signature then implies that

$$
\sigma(Z)=-8-(2 g+10) \sigma(Y)=4(-2-(n+3) \sigma(Y)),
$$

where (as before) $g=2 n+1$. Now the signature $\sigma(Z)$ is not zero; in fact, since $\sigma(Y)$ is nonzero, $|\sigma(Z)|$ is of the magnitude of $2 g|\sigma(Y)|$.

In [16], examples of surface bundles over surfaces of genus 9 with $\sigma=4$ (and fiber genus at least 3 ) have been constructed. The signatures in [16] were computed by more algebraic methods, relying on Meyer's signature cocycle [36]. The result of Bryan and Donagi [8] provides the surface bundle with nonvanishing signature and the smallest possible base genus: a genus- 25 fibration over a genus- 2 surface with signature 16. (Since $\sigma+\chi$ is divisible by 4 and $\chi$ for a surface bundle over a surface is $4(g-1)(h-1)$, it follows that the signatures of such manifolds are always divisible by 4 .)

The significance of surface bundles also comes from the fact that, once the fiber genus is at least 2, these manifolds admit symplectic structures with both orientations. In particular, once $b_{2}^{+}>1$, this property implies that these manifolds admit nontrivial Seiberg-Witten invariants with both orientations.

Assume that $X$ is the total space of a surface bundle over a surface with fiber genus at least 2 . Then $X$ is minimal (as a symplectic manifold): Indeed, by choosing an almost complex structure $J$ for which the bundle map is $J$-holomorphic, any -1 -sphere would give rise to a $(-1)$-sphere in a fiber; by Remark 2.6 the -1 -sphere can be assumed to be $J$-holomorphic, hence the bundle map restricted to the sphere gives a holomorphic map from $S^{2}$ to the base complex curve (of positive genus), and this map must be constant (so $S^{2}$ is in a fiber). By Taubes' theorem this property and $b_{2}^{+}(X)>1$ implies that $c_{1}^{2}(X)=3 \sigma(X)+2 \chi(X) \geq 0$. Since this inequality applies for both orientations (while $\sigma(-X)=-\sigma(X)$ and $\chi(-X)=\chi(X)$ ), we get

$$
3|\sigma(X)| \leq 2 \chi(X) .
$$

This inequality has been improved by Kotschick [28]:

Proposition 7.1 (Kotschick [28]) Suppose that $X$ admits a surface bundle structure with base and fiber genera at least 2 . Then

$$
2|\sigma(X)| \leq \chi(X) .
$$

Proof Notice that by Theorem $3.4 \mathrm{X}$ with either orientation is a symplectic manifold (since the assumption on the fiber genus guarantees that the fiber is homologically essential). As above, it can be shown that the manifold $X$ is minimal, that is, does not 
contain any embedded sphere with self-intersection -1 . It can be shown that $b_{2}^{+}>1$ holds for both orientations, hence $c_{1}^{2}(X) \geq 0$ and $c_{1}^{2}(-X) \geq 0$ (where $-X$ denotes $X$ with the reversed orientation).

By Taubes' theorem we can consider a $J$-holomorphic representative $C \subset X$ of $-c_{1}(X, \omega)$, where $\omega$ is a symplectic form given by the fibration structure and $J$ is an $\omega$-compatible almost complex structure. Suppose that $C$ is connected. (Taubes' result does not provide connected representatives, and indeed, sometimes the $J$-holomorphic representative cannot be chosen to be connected; in the argument to come we assume connectedness just to simplify the presentation.) Suppose that the intersection number of $C$ with the typical fiber $F$ is $d$. Then the restriction of the bundle map $f$ to $C$ provides a $d$-fold branched cover $f_{C}: C \rightarrow \Sigma_{h}$. By Kneser's formula, this implies

$$
g(C)-1 \geq d(h-1)
$$

By the adjunction formula for the generic fiber $F$ (and by the fact that $F \cdot F=0$ ), we get that

$$
C \cdot F=2 g(F)-2 \text {. }
$$

Since $d=C \cdot F$, the above two formulae show that $g(C)-1 \geq \frac{1}{2} \chi(X)$. Applying the adjunction equality now for $C$ we get

$$
\frac{1}{2} \chi(X) \leq g(C)-1=\frac{1}{2}\left(C \cdot C-c_{1}(X, \omega) \cdot C\right)=C \cdot C=3 \sigma(X)+2 \chi(X) .
$$

This argument shows that $\chi(X) \geq-2 \sigma(X)$; this inequality together with the similar one for $-X$ implies the claimed inequality of the proposition.

The above result has been recently improved by Hamenstädt:

Theorem 7.2 (Hamenstädt [22]) Suppose that $X$ is a 4-manifold with a surface bundle map $f: X \rightarrow \Sigma_{h}$. Then

$$
3|\sigma(X)| \leq \chi(X)
$$

Recall that for a compact complex surface $X$ of general type we have the famous Bogomolov-Miyaoka-Yau (BMY) inequality

$$
c_{1}^{2}(X) \leq 3 c_{2}(X)
$$

This inequality is sharp in the sense that there are complex surfaces having characteristic numbers with equality in Equation (7-1). In fact, by a result of LeBrun [31], this inequality holds for those symplectic manifolds which admit an Einstein metric. It is 
easy to see that for a surface bundle $X \rightarrow \Sigma_{h}$, the inequality $3 \sigma(X) \leq \chi(X)$ (following from Hamenstädt's Theorem 7.2) implies

$$
c_{1}^{2}(X)=3 \sigma(X)+2 \chi(X) \leq 3 \chi(X)=3 c_{2}(X),
$$

providing the BMY inequality for surface bundles.

This connection then brings us to one of the most intriguing questions in 4-dimensional symplectic topology:

Problem 7.3 Prove or provide a counterexample to the BMY inequality (7-1) for 4-manifolds admitting a Lefschetz fibration structure. More generally, prove or provide a counterexample to the BMY inequality (7-1) for closed symplectic 4-manifolds.

\section{Stein surfaces and Stein domains}

The method of considering Lefschetz fibrations and Lefschetz pencils on closed 4manifolds (hence providing a bridge between symplectic structures and mapping class groups) extends to 4-manifolds with boundary. In order to state the relevant result, we need to review a central concept from complex geometry.

Suppose that $V$ is a complex manifold. It is a Stein manifold if $V$ admits a proper, holomorphic embedding into $\mathbb{C}^{n}$ for some $n$. From this perspective, Stein manifolds are the natural complex counterparts of (real) manifolds, which (by the Whitney embedding theorem) all admit embedding into some $\mathbb{R}^{m}$. From the maximum principle it follows that a Stein manifold is necessarily noncompact.

An alternative definition (relying more on the concept of symplectic forms) can be given as follows. Suppose that $(V, J)$ is a complex manifold with complex structure $J$. Suppose that $\varphi: V \rightarrow[0, \infty)$ is a proper, smooth function on $V$. Consider the 1 -form $d \varphi$, and define $d^{\mathbb{C}} \varphi$ by the formula

$$
d^{\mathbb{C}} \varphi(v)=d \varphi(J v) .
$$

Let $\omega_{\varphi}$ denote the 2-form $-d\left(d^{\mathbb{C}} \varphi\right)$.

Definition 8.1 The function $\varphi$ with the above properties is strictly plurisubharmonic (spsh) if the associated $2-$ form $\omega_{\varphi}$ is nondegenerate, consequently is a symplectic form on $V$. The triple $(V, J, \varphi)$ is a Stein manifold if $(V, J)$ is a complex manifold and $\varphi$ is a spsh function. 
(Notice that the 2 -form $\omega_{\varphi}$ is exact, hence it is symplectic if and only if it is nondegenerate. Also, if $\omega_{\varphi}$ is an exact symplectic form, then $V$ is necessarily noncompact.) It is a classical result that the two definitions of Stein manifolds described above are equivalent.

A compact 4-manifold $X$ with boundary $Y$ and complex structure $J$ on $X-Y$ is a Stein domain if there is a Stein manifold $(V, J, \varphi)$ and a regular value $c$ of $\varphi$ such that $\varphi^{-1}([0, c])$ and $(X, J)$ are biholomorphic.

A striking result of Eliashberg $[11 ; 14]$ describes Stein manifolds and Stein domains in dimensions at least 6 by a convenient topological property: the (real) $2 n$-dimensional manifold $V$ admits a Stein structure if and only if

- $\quad V$ admits an almost complex structure, and

- $\quad V$ admits a proper Morse function $V \rightarrow[0, \infty)$ which has critical points only of index $\leq n$.

Such a characterization of Stein 4-manifolds is not available; both conditions are necessary, but (as the example of $S^{2} \times D^{2}$ shows) are not sufficient. The further condition one needs to build a Stein structure on an oriented 2-handlebody (ie a 4manifold built from $0-, 1-$ and 2-handles only) is in the attachment of the 2-handles. (Notice that an oriented 2-handlebody always admits an almost complex structure.) In fact, the boundary of the union of $0-$ and 1-handles admits a canonical (tight) contact structure, and we need that each 2 -handle can be attached along a Legendrian knot, with framing one less than the contact framing of the knot. This condition is rather delicate, and therefore it is a challenging problem in general to decide whether a 4-manifold admits a Stein structure, or even if a 3-manifold bounds a Stein domain.

Seiberg-Witten theory, and arguments based on these invariants provide strong tools to understand topological properties of Stein 4-manifolds. A prominent example of such results is the following theorem:

Theorem 8.2 (Lisca and Matić [33]) Suppose that $\Sigma \subset X$ is a closed, oriented surface of genus $g(\Sigma)$ in a Stein domain $(X, J)$ with first Chern class $c_{1}(X, J)$. Then

$$
\left|\left\langle c_{1}(X, J),[\Sigma]\right\rangle\right|+[\Sigma]^{2} \leq 2 g(\Sigma)-2 .
$$

Notice, in particular, that the above inequality implies that a Stein domain does not contain an embedded sphere with self-intersection -1 .

It is now rather easy to see that some 4-manifolds cannot carry Stein structures: for example, the positive-definite $E_{8}$ plumbing $X_{E_{8}}$ (containing many spheres with 
self-intersection +2 ) cannot be made Stein. It requires a more advanced argument to show, that, in fact its boundary $Y_{E_{8}}=\partial X_{E_{8}}$ is not the boundary of any Stein domain. This result (due to Lisca [32]) involves two steps: Using an advanced version of Seiberg-Witten theory one can show that any Stein domain with boundary $Y_{E_{8}}$ must have negative definite intersection form. Then gluing a potential Stein domain $Z$ with $\partial Z=Y_{E_{8}}$ to $-X_{E_{8}}$ (the "negative-definite $E_{8}$-plumbing") we get a negativedefinite closed 4-manifold $W$ with its intersection form containing the negative-definite $E_{8}$ lattice. Since by Donaldson's famous theorem this 4 -manifold $W$ must have diagonalizable intersection form (diagonalizable over $\mathbb{Z}$ ), while the negative-definite $E_{8}$ lattice does not embed into any diagonal lattice over $\mathbb{Z}$ (as a rather elementary argument shows), we find a contradiction from the assumption of the existence of $Z$.

This shows that the property whether a 3-manifold is the boundary of a Stein domain is far from being transparent. (This question has been studied for higher-dimensional manifolds as well [6]; in dimension at least 5, based on Eliashberg's theorem, surgerytheoretic methods can be fruitfully applied, while the complete picture for 3-manifolds is still rather mysterious.)

\section{Stein domains and Lefschetz fibrations}

Examples of Stein domains can be given by the construction from the beginning of Section 4. In fact, by considering the same situation of having a complex surface $X \subset \mathbb{C P}^{n}$, let us take a hyperplane $\mathbb{C P}^{n-1} \subset \mathbb{C P}^{n}$ and define $V$ as the intersection

$$
X \cap\left(\mathbb{C P}^{n}-\mathbb{C P}^{n-1}\right) .
$$

Since the complement of the hyperplane in $\mathbb{C P}^{n}$ is $\mathbb{C}^{n}$, the above intersection admits the required embedding into $\mathbb{C}^{n}$, hence is a Stein surface. Recall that a family of hyperplanes (containing the fixed axis $A$ ) provides a Lefschetz pencil structure on $X$. Indeed, this pencil provides a fibration on $X \cap\left(\mathbb{C P}^{n}-\mathbb{C P}^{n-1}\right)$ : by deleting $X \cap \mathbb{C P}^{n-1}$ we delete all the base points of the pencil, hence we get a Lefschetz fibration

$$
f: X-\left(X \cap \mathbb{C P}^{n-1}\right) \longrightarrow D^{2}
$$

over the 2-disk $D^{2}$. The fiber of this fibration is given by deleting $X \cap \mathbb{C P}^{n-1}$ from a curve of the pencil, hence we get a 2 -dimensional surface with nonempty boundary. This leads us to the following definition:

Definition 9.1 Suppose that $X$ is a smooth, compact, oriented 4-manifold with nonempty boundary $\partial X=Y$ and $\Sigma$ is a compact 2-dimensional manifold with 
nonempty boundary. The smooth map $f: X \rightarrow \Sigma$ is a Lefschetz fibration with bounded fibers if $d f$ is onto with finitely many exceptions $C=\left\{p_{1}, \ldots, p_{k}\right\}, C$ is contained in the interior $X-\partial X$ and at a point of $C$ we have Lefschetz charts just as in Definition 3.1.

The above complex geometric construction provides such a fibration structure for any $X-\left(X \cap \mathbb{C P}^{n-1}\right)$ (where $X \subset \mathbb{C P}^{n}$ is a projective surface). In fact, in these cases the base surface is simply the 2-disk $D^{2}$.

Donaldson's Theorem 3.3, extending the existence of a Lefschetz pencil from projective surfaces to symplectic 4-manifolds, extends the above construction as well: suppose that $(X, \omega)$ is a closed symplectic 4 -manifold. Then the appropriate Lefschetz pencil of Theorem 3.3 provides a Stein structure on the complement of the generic curve of the pencil, and also equips this complement with a Lefschetz fibration with bounded fibers. These examples, however, do not exhaust all Stein 4-manifolds; for example, the boundary of a Stein manifold constructed in this way is a circle bundle over a surface, while there are Stein domains with different boundaries. Nevertheless, the fibration structure and the Stein structure on a 4-manifold are closely related:

Theorem 9.2 (Loi and Piergallini [35], Akbulut and Ozbagci [1]) A Stein domain $X$ admits a Lefschetz fibration over $D^{2}$ with bounded fibers. The genus of the fiber can be chosen to be arbitrarily large. Conversely, the total space of a Lefschetz fibration over $D^{2}$ with bounded fibers and with only homologically essential vanishing cycles admits a Stein structure.

This result admits an extension to Lefschetz fibrations over surfaces other than $D^{2}$ :

Theorem 9.3 (Lisi and Wendl, Baykur and Van Horn-Morris [3]) Suppose that the 4-manifold with boundary admits a Lefschetz fibration $f: X \rightarrow \Sigma$ with bounded fibers over the 2-manifold $\Sigma$ with nonempty boundary. If the vanishing cycles corresponding to the Lefschetz singularities are all homologically essential, then $X$ admits a Stein structure.

Remark 9.4 It is clear that some assumption on the vanishing cycles is needed, since a Stein domain is minimal (in the sense that it contains no sphere of self-intersection -1 , cf the adjunction formula in Theorem 8.2), while a homotopically trivial vanishing cycle produces an embedded sphere of self-intersection -1 . While the above result has been known when the base surface $\Sigma$ is diffeomorphic to the disk for quite some time, this general form was proved only recently. 


\section{Stein domains and mapping class groups}

Just as in the closed case, we can apply mapping class group techniques to encode bounded Lefschetz fibrations. Indeed, suppose that $f: X \rightarrow \Sigma$ is a bounded Lefschetz fibration with fiber of genus $g$ and $r$ boundary components. As in the description of Lefschetz pencils, the monodromies along loops in $\Sigma$ avoiding critical values provide elements of the mapping class group $\Gamma_{g}^{r}$ (that is, mapping classes which keep the boundary of the fiber pointwise fixed). Consequently, a Lefschetz fibration with bounded fibers over $D^{2}$ gives rise to a product

$$
\prod_{i=1}^{k} t_{i} \in \Gamma_{g}^{r}
$$

of right-handed Dehn twists in the mapping class group $\Gamma_{g}^{r}$; if the fibration is over a genus- $m$ surface $\Sigma$, then (again after the choice of a generating system of curves $a_{j}, b_{j}$ in $\Sigma$ for $\left.\pi_{1}(\Sigma)\right)$ we get, as the corresponding monodromies,

$$
\prod_{i=1}^{k} t_{i} \prod_{j=1}^{m}\left[\alpha_{j}, \beta_{j}\right] \in \Gamma_{g}^{r} .
$$

Unlike for Lefschetz pencils in the closed case, now we cannot be sure that these products factor the product of Dehn twists along boundary parallel curves. Indeed, the product above gives rise to an element $h \in \Gamma_{g}^{r}$, which equips the boundary 3manifold with an extra structure: an open book in case $\Sigma=D^{2}$ and a spinal open book in general (for a detailed discussion on spinal open books see the forthcoming paper of Lisi, Van Horn-Morris and Wendl [34], or [3, Appendix]). Here we will not discuss these structures in any further detail; we restrict ourselves to the comment that (spinal) open books provide the mapping class group analogue of contact structures on 3-manifolds.

As in the closed case, we are interested in the number of singular fibers a Lefschetz fibration can admit. Notice that (since the open book, and hence the monodromy, on the boundary can be arbitrary) the factorization can be arbitrarily short: by considering the 3-manifold admitting the open book decomposition with monodromy $h$ being equal to a right-handed Dehn twist, the factorization can be of length one. The question therefore makes sense only after an open book decomposition (or equivalently, an element $h \in \Gamma_{g}^{r}$ ) is fixed. Let us consider first the special case when $h=1$.

Proposition 10.1 [41] Suppose that $\prod_{i=1}^{k} t_{i}$ is the product of right-handed Dehn twists with the property that $\prod_{i=1}^{k} t_{i}=1 \in \Gamma_{g}^{r}$ and $r>0$. Then $k=0$ and the product is the empty product. 
Proof Suppose that we have a nontrivial factorization of 1. Considering it in $\Gamma_{g}$, we get a nontrivial Lefschetz fibration over the sphere, and since it lifts to $\Gamma_{g, r}$, it admits sections. Since the lift gives 1 in $\Gamma_{g}^{r}$, these sections have 0 self-intersection, contradicting Proposition 5.8.

This result shows that we cannot increase the length of a factorization by multiplying it with the factorization of the unit element. (Such increase of the length of a factorization does work in the case when $r=0$, and indeed it corresponds to fiber summing Lefschetz fibrations.) This observation led to the expectation that perhaps the factorization of a fixed element $h \in \Gamma_{g}^{r}$ is bounded from above.

This boundedness conjecture (proved for further special monodromies besides $1 \in \Gamma_{g}^{r}$ in [43]) has been recently disproved by Baykur and Van Horn-Morris in [4] (see also [3]):

Theorem 10.2 (Baykur and Van Horn-Morris [4]) Suppose that $\Sigma$ is a genus- $g$ surface with two boundary components and with $g \geq 8$. Then the product $\delta_{1} \delta_{2}$ can be factorized as a product of arbitrarily large number of positive Dehn twists along nonseparating curves in $\Gamma_{g}^{2}$.

Since the number of singular fibers (or equivalently, the length of the factorization of the monodromy) is directly linked to the Euler characteristic of the Stein filling, the above result provided sequences of Stein manifolds with fixed boundary and with growing Euler characteristic. Theorem 10.2 has been extended to lower-genus surfaces:

Theorem 10.3 (Dalyan, Korkmaz and Pamuk [12]) Let $\Sigma_{g}^{1}$ be the compact connected oriented surface of genus $g$ with one boundary component $\delta$. In the mapping class group $\Gamma_{g}^{1}$ consider the element $h$ which is equal to $\delta^{2}$ for $g=2$ and $\delta$ for $g \geq 3$. Then $h$ can be written as a product of arbitrarily large number of right-handed Dehn twists along nonseparating simple closed curves.

\section{References}

[1] S Akbulut, B Ozbagci, Lefschetz fibrations on compact Stein surfaces, Geom. Topol. 5 (2001) 319-334 MR1825664

[2] MF Atiyah, The signature of fibre-bundles, from: "Global Analysis", Univ. Tokyo Press, Tokyo (1969) 73-84 MR0254864

[3] R I Baykur, J Van Horn-Morris, Families of contact 3-manifolds with arbitrarily large Stein fillings, preprint (2012) arXiv:1208.0528

[4] R I Baykur, J Van Horn-Morris, Topological complexity of symplectic 4-manifolds and Stein fillings, preprint (2012) arXiv:1212.1699 
[5] M Bestvina, K Fujiwara, Bounded cohomology of subgroups of mapping class groups, Geom. Topol. 6 (2002) 69-89 MR1914565

[6] J Bowden, D Crowley, A I Stipsicz, The topology of Stein fillable manifolds in high dimensions, I, Proc. Lond. Math. Soc. 109 (2014) 1363-1401 MR3293153

[7] V Braungardt, D Kotschick, Clustering of critical points in Lefschetz fibrations and the symplectic Szpiro inequality, Trans. Amer. Math. Soc. 355 (2003) 3217-3226 MR1974683

[8] J Bryan, R Donagi, Surface bundles over surfaces of small genus, Geom. Topol. 6 (2002) 59-67 MR1885589

[9] J Bryan, R Donagi, A I Stipsicz, Surface bundles: Some interesting examples, Turkish J. Math. 25 (2001) 61-68 MR1829079

[10] S S Chern, F Hirzebruch, J-P Serre, On the index of a fibered manifold, Proc. Amer. Math. Soc. 8 (1957) 587-596 MR0087943

[11] K Cieliebak, Y Eliashberg, From Stein to Weinstein and back: Symplectic geometry of affine complex manifolds, Amer. Math. Soc. Colloq. Pub. 59, Amer. Math. Soc. (2012) MR3012475

[12] E Dalyan, M Korkmaz, M Pamuk, Arbitrarily long factorizations in mapping class groups, preprint (2014) arXiv:1309.3778

[13] S K Donaldson, Lefschetz pencils on symplectic manifolds, J. Differential Geom. 53 (1999) 205-236 MR1802722

[14] Y Eliashberg, Topological characterization of Stein manifolds of dimension > 2, Internat. J. Math. 1 (1990) 29-46 MR1044658

[15] H Endo, A construction of surface bundles over surfaces with non-zero signature, Osaka J. Math. 35 (1998) 915-930 MR1659565

[16] H Endo, M Korkmaz, D Kotschick, B Ozbagci, A Stipsicz, Commutators, Lefschetz fibrations and the signatures of surface bundles, Topology 41 (2002) 961-977 MR1923994

[17] H Endo, D Kotschick, Bounded cohomology and non-uniform perfection of mapping class groups, Invent. Math. 144 (2001) 169-175 MR1821147

[18] B Farb, D Margalit, A primer on mapping class groups, Princeton Mathematical Series 49, Princeton Univ. Press (2012) MR2850125

[19] R E Gompf, The topology of symplectic manifolds, Turkish J. Math. 25 (2001) 43-59 MR1829078

[20] R E Gompf, Toward a topological characterization of symplectic manifolds, J. Symplectic Geom. 2 (2004) 177-206 MR2108373

[21] R E Gompf, A I Stipsicz, 4-manifolds and Kirby calculus, Graduate Studies in Mathematics 20, Amer. Math. Soc. (1999) MR1707327 
[22] U Hamenstaedt, Signatures of surface bundles and Milnor Wood inequalities, preprint (2012) arXiv:1206.0263

[23] J A Hillman, Sections of surface bundles, preprint (2014) arXiv:1309.3803

[24] F Hirzebruch, H Hopf, Felder von Flächenelementen in 4-dimensionalen Mannigfaltigkeiten, Math. Ann. 136 (1958) 156-172 MR0100844

[25] K Kodaira, A certain type of irregular algebraic surfaces, J. Analyse Math. 19 (1967) 207-215 MR0216521

[26] M Korkmaz, Stable commutator length of a Dehn twist, Michigan Math. J. 52 (2004) 23-31 MR2043394

[27] M Korkmaz, B Ozbagci, Minimal number of singular fibers in a Lefschetz fibration, Proc. Amer. Math. Soc. 129 (2001) 1545-1549 MR1713513

[28] D Kotschick, Signatures, monopoles and mapping class groups, Math. Res. Lett. 5 (1998) 227-234 MR1617905

[29] P B Kronheimer, T S Mrowka, Gauge theory for embedded surfaces, I, Topology 32 (1993) 773-826 MR1241873

[30] P B Kronheimer, T S Mrowka, The genus of embedded surfaces in the projective plane, Math. Res. Lett. 1 (1994) 797-808 MR1306022

[31] C LeBrun, Einstein metrics and Mostow rigidity, Math. Res. Lett. 2 (1995) 1-8 MR1312972

[32] P Lisca, Symplectic fillings and positive scalar curvature, Geom. Topol. 2 (1998) 103-116 MR1633282

[33] P Lisca, G Matić, Stein 4-manifolds with boundary and contact structures, Topology Appl. 88 (1998) 55-66 MR1634563

[34] S Lisi, J Van Horn-Morris, C Wendl, On symplectic fillings of spinal open book decompositions in preparation

[35] A Loi, R Piergallini, Compact Stein surfaces with boundary as branched covers of $B^{4}$, Invent. Math. 143 (2001) 325-348 MR1835390

[36] W Meyer, Die Signatur von Flächenbündeln, Math. Ann. 201 (1973) 239-264 MR0331382

[37] J W Morgan, The Seiberg-Witten equations and applications to the topology of smooth four-manifolds, Mathematical Notes 44, Princeton Univ. Press (1996) MR1367507

[38] L I Nicolaescu, Notes on Seiberg-Witten theory, Graduate Studies in Mathematics 28, Amer. Math. Soc. (2000) MR1787219

[39] B Siebert, G Tian, On the holomorphicity of genus-two Lefschetz fibrations, Ann. of Math. 161 (2005) 959-1020 MR2153404

[40] A I Stipsicz, On the number of vanishing cycles in Lefschetz fibrations, Math. Res. Lett. 6 (1999) 449-456 MR1713143 
[41] A I Stipsicz, Sections of Lefschetz fibrations and Stein fillings, Turkish J. Math. 25 (2001) 97-101 MR1829081

[42] A I Stipsicz, Singular fibers in Lefschetz fibrations on manifolds with $b_{2}^{+}=1$, Topology Appl. 117 (2002) 9-21 MR1874001

[43] A I Stipsicz, On the geography of Stein fillings of certain 3-manifolds, Michigan Math. J. 51 (2003) 327-337 MR1992949

[44] C H Taubes, The Seiberg-Witten invariants and symplectic forms, Math. Res. Lett. 1 (1994) 809-822 MR1306023

[45] C H Taubes, SW $\Rightarrow$ Gr: From the Seiberg-Witten equations to pseudo-holomorphic curves, J. Amer. Math. Soc. 9 (1996) 845-918 MR1362874

[46] E Witten, Monopoles and four-manifolds, Math. Res. Lett. 1 (1994) 769-796 MR1306021

Rényi Institute of Mathematics, Hungarian Academy of Sciences

H-1053 Budapest, Reáltanoda utca 13-15, Hungary

stipsicz.andras@renyi.mta.hu

Received: 28 January 2015 